\title{
DIRICHLET PROBLEM AND SOKHOTSKI-PLEMELJ JUMP FORMULA ON WEIL-PETERSSON CLASS QUASIDISKS
}

\author{
David Radnell, Eric Schippers and Wolfgang Staubach \\ Aalto University, Department of Mathematics and Systems Analysis \\ P. O. Box 11100, FI-00076 Aalto, Finland; david.radnell@aalto.fi \\ University of Manitoba, Department of Mathematics \\ Winnipeg, Manitoba, R3T 2N2, Canada; eric_schippers@umanitoba.ca \\ Uppsala University, Department of Mathematics \\ Box 480, 75106 Uppsala, Sweden; wulf@math.uu.se
}

\begin{abstract}
We show the solvability of the Dirichlet problem on Weil-Petersson class quasidisks and establish a Sokhotski-Plemelj jump formula for Weil-Petersson class quasicircles. Furthermore we show that the resulting Cauchy projections are bounded. In both cases the boundary data belongs to a certain conformally invariant Besov space. Moreover we show that the WP-class quasicircles are chord-arc curves.
\end{abstract}

\section{Introduction}

In this paper we demonstrate that the Dirichlet problem is solvable on a WPclass quasidisk $\Omega$ with boundary values in a certain Besov space $\mathcal{H}(\partial \Omega)$, and that traces of finite Dirichlet energy harmonic functions are in this Besov space. Thus we obtain a precise characterization of the set of boundary values of complex harmonic functions of finite Dirichlet energy for WP-class quasidisks. We also show that this Besov space is conformally invariant in the sense that composition by a conformal map onto another WP-class quasidisk is a bounded invertible map. We also extend the Sokhotski-Plemelj jump decomposition to $\mathcal{H}(\Gamma)$ on any Weil-Petersson class quasicircle $\Gamma$. The resulting Cauchy projections are bounded. In the case that $\Gamma=\mathbf{S}^{1}$, $\mathcal{H}(\Gamma)$ becomes the Sobolev space of function with square-integrable half-order derivatives. The WP-class quasicircles are a strict subclass of the so-called asymptotically conformal quasicircles, which have been an object of interest for the last decade. Their study was initiated by Cui [5] and Guo [9], in connection with finding a theory of the universal Teichmüller space based on $L^{p}$ Beltrami differentials and conformal maps. The memoir [18] of Takhtajan and Teo obtained wide-ranging results on the WP-class universal Teichmüller space, including for example sewing formulas for the Laplacian and potentials for the Weil-Petersson metric. This stimulated a great deal of interest in the subject (see Shen [15]). The analytic problems in this paper have applications to a function-theoretic model of the Weil-Petersson class Teichmüller space of Riemann surfaces of genus zero with $n$ boundary curves, and also to the

doi: $10.5186 /$ aasfm. 2016.4108

2010 Mathematics Subject Classification: Primary 30F15, 31C05, 31C25; Secondary 30C55, $30 \mathrm{C} 62$.

Key words: Dirichlet problem, quasicircles, quasiconformal extension, Poincaré inequality, chord-arc curves, Sokhotski-Plemelj jump decomposition, Cauchy integral, Besov spaces, WeilPetersson class. 
existence of determinants of certain elliptic operators in conformal field theory [10, Appendix D]. These results will appear in two future publications.

The analysis in the paper involves Sobolev and Dirichlet spaces on quasidisks. In particular we require some results of of Smith and Stegenga [16] and Stanoyevitch and Stegenga [17] concerning Poincaré inequalities on John domains. Later in the investigation of the regularity of the WP-class quasicircles we utilize a result from geometric measure theory due to Falconer and Marsh [7] characterizing bi-Lipschitz equivalence of quasidisks. Furthermore the study of Sobolev and Besov spaces, and in particular the traces of functions on the boundary of so called Ahlfors-regular domains (to which we show the WP-class quasidisks belong), was investigated by Jonsson [11]. Finally the existence of the solution to the Dirichlet problem on the WP-class quasidisks, with Besov space boundary data, hinges on results of Chang and Lewis [4] and Mitrea, Mitrea and Monniaux [13].

\section{Dirichlet problem}

In this section we show the solvability of the Dirichlet problem with values in a certain Besov space. This is done by using some known facts and results from geometric measure theory and elliptic PDEs and combining these with our function theoretic lemmas and theorems. In particular we characterize the boundary values of harmonic functions of finite Dirichlet energy.

2.1. Function spaces associated with the Dirichlet problem. Let us first recall the definition of the holomorphic Dirichlet spaces. Let $\Omega$ be a simplyconnected domain in the Riemann sphere $\overline{\mathbf{C}}$. We will assume that 0 and $\infty$ are not on the boundary of $\Omega$. The Dirichlet space is

$$
\mathcal{D}(\Omega)=\left\{h: \Omega \rightarrow \mathbf{C}: h \text { holomorphic in } \Omega \text { and } \iint_{\Omega}\left|h^{\prime}\right|^{2} d A<\infty\right\},
$$

if $\infty \notin \Omega$, and if $\infty \in \Omega$, then

$$
\mathcal{D}(\Omega)=\left\{h: \Omega \rightarrow \mathbf{C}: h \text { holomorphic in } \Omega, h(\infty)=0 \text { and } \iint_{\Omega}\left|h^{\prime}\right|^{2} d A<\infty\right\} .
$$

We endow $\mathcal{D}(\Omega)$ with the norm

$$
\|h\|=\left(\iint_{\Omega}\left|h^{\prime}\right|^{2} d A\right)^{1 / 2}
$$

if $\infty \in \Omega$, and with the norm

$$
\|h\|=\left(|h(0)|^{2}+\iint_{\Omega}\left|h^{\prime}\right|^{2} d A\right)^{1 / 2}
$$

if $0 \in \Omega$. The convention that functions in $\mathcal{D}(\Omega)$ satisfy $h(\infty)=0$ if $\infty$ is in $\Omega$ is a matter of convenience, which we adopt because functions obtained by a Cauchy integral will have this property.

We will also require the space of harmonic functions of finite Dirichlet energy on a quasidisk, and the characterization of their boundary values. Here for definiteness we assume that $0 \in \Omega$ and $\infty$ is not in the closure of $\Omega$. Other cases can be obtained by composition with a Möbius transformation. We define the harmonic Dirichlet 
space

$$
\begin{aligned}
& \mathcal{D}_{\text {harm }}(\Omega)=\{h: \Omega \rightarrow \mathbf{C}: h \text { harmonic in } \Omega \text { and } \\
&\left.\qquad \iint_{\Omega}\left|h^{\prime}\right|^{2} d A+\iint_{\Omega}\left|\bar{h}^{\prime}\right|^{2} d A<\infty\right\} .
\end{aligned}
$$

We endow $\mathcal{D}_{\text {harm }}(\Omega)$ with a norm

$$
\|h\|_{\mathcal{D}_{\text {harm }}(\Omega)}=\left\{|h(0)|^{2}+\iint_{\Omega}\left|h^{\prime}\right|^{2} d A+\iint_{\Omega}\left|\bar{h}^{\prime}\right|^{2} d A\right\}^{\frac{1}{2}} .
$$

Next we recall the definition of Sobolev spaces on open connected subsets of $\mathbf{R}^{2}$.

Definition 2.1. Let $\Omega$ be an open connected domain in the plane. Denote by $H^{1}(\Omega)$ the Sobolev space of functions in $L^{2}(\Omega)$ with

$$
\|h\|_{H^{1}(\Omega)}:=\left\{\|h\|_{L^{2}(\Omega)}^{2}+\left\|h^{\prime}\right\|_{L^{2}(\Omega)}^{2}+\left\|\bar{h}^{\prime}\right\|_{L^{2}(\Omega)}^{2}\right\}^{\frac{1}{2}}<\infty,
$$

where the derivations are in the sense of distributions.

Theorem 2.2. Let $\Omega$ be a quasidisk containing 0 . Then for $h \in \mathcal{D}_{\text {harm }}(\Omega)$ one has

$$
C^{\prime}\|h\|_{\mathcal{D}_{\text {harm }}} \leq\|h\|_{H^{1}(\Omega)} \leq C\|h\|_{\mathcal{D}_{\text {harm }}}
$$

Proof. To show that $\|h\|_{H^{1}(\Omega)} \leq C\|h\|_{\mathcal{D}_{\text {harm }}}$, using (2.6) and (2.7), it would be enough to show that $\|h\|_{L^{2}(\Omega)}^{2} \leq C\left(|h(0)|^{2}+\left\|h^{\prime}\right\|_{L^{2}(\Omega)}^{2}+\left\|\bar{h}^{\prime}\right\|_{L^{2}(\Omega)}^{2}\right)$, for $h \in \mathcal{D}_{\text {harm }}(\Omega)$.

Now it is well-known, see for example [16] and [17], that for any quasidisk $\Omega$ (which is a so called John domain), for any arbitrary $z_{0}$ in $\Omega$, and for $F$ holomorphic in $\Omega$, one has the analytic Poincaré inequality

$$
\left\|F-F\left(z_{0}\right)\right\|_{L^{2}(\Omega)} \leq C\left(z_{0}\right)\left\|F^{\prime}\right\|_{L^{2}(\Omega)} .
$$

Now since $\Omega$ is simply connected, any $h \in \mathcal{D}_{\text {harm }}(\Omega)$ can be represented as $h(z)=$ $F(z)+\overline{G(z)}$, where $F$ and $G$ are holomorphic functions in $\Omega$. Therefore, (2.9) yields

$$
\begin{aligned}
\|h\|_{L^{2}(\Omega)}^{2} & \leq 2\|h-h(0)\|_{L^{2}(\Omega)}^{2}+2|\Omega \| h(0)|^{2} \\
& \leq 4\|F-F(0)\|_{L^{2}(\Omega)}^{2}+4\|G-G(0)\|_{L^{2}(\Omega)}^{2}+2|\Omega \| h(0)|^{2} \\
& \leq 2|\Omega||h(0)|^{2}+C_{1}\left(\left\|F^{\prime}\right\|_{L^{2}(\Omega)}^{2}+\left\|G^{\prime}\right\|_{L^{2}(\Omega)}^{2}\right) \\
& \leq C_{2}\left(|h(0)|^{2}+\left\|h^{\prime}\right\|_{L^{2}(\Omega)}^{2}+\left\|\bar{h}^{\prime}\right\|_{L^{2}(\Omega)}^{2}\right),
\end{aligned}
$$

where we have also used the holomorphicity of $F$ and $G$ which yields that $F^{\prime}(z)=$ $h^{\prime}(z)$ and $G^{\prime}(z)=\bar{h}^{\prime}$. This concludes the proof of the second estimate in (2.8).

In order to show $C^{\prime}\|h\|_{\mathcal{D}_{\text {harm }}} \leq\|h\|_{H^{1}(\Omega)}$, it is enough to show that $|h(0)|^{2} \leq$ $C\|h\|_{L^{2}(\Omega)}^{2}$. To this end, we observe that since $0 \in \Omega$ and $h$ is harmonic in $\Omega$, there is an $r>0$ such that $\mathbf{D}(0, r) \subset \Omega$ and by the mean-value theorem for harmonic functions one has

$$
|h(0)| \leq \frac{1}{\pi r^{2}} \iint_{\mathbf{D}(0, r)}|h(z)| d A(z) \leq \frac{|\Omega|}{\pi r^{2}} \iint_{\Omega}|h(z)| \frac{d A(z)}{|\Omega|} .
$$

Now Jensen's inequality yields that

$$
|h(0)|^{2} \leq \frac{|\Omega|}{\pi^{2} r^{4}} \iint_{\Omega}|h(z)|^{2} d A(z)
$$

which gives us the desired estimate. This ends the proof of the theorem. 
2.2. WP-class quasidisks and their regularity. Let $\mathbf{D}^{+}=\{z:|z|<1\}$ and $\mathbf{D}^{-}=\{z:|z|>1\} \cup\{\infty\}$. Denote the set of Beltrami differentials on $\mathbf{D}^{-}$satisfying

$$
\iint_{\mathbf{D}^{-}} \frac{|\mu(z)|^{2}}{\left(|z|^{2}-1\right)^{2}} d A<\infty
$$

by $L_{\text {hyp }}^{2}\left(\mathbf{D}^{-}\right)$("hyp" stands for "hyperbolic", since the condition above says that $|\mu|$ is square-integrable with respect to the hyperbolic area element on $\mathbf{D}^{-}$). With this in mind, we make the following definition, following terminology of [14].

Definition 2.3. Let $f: \mathbf{D}^{+} \rightarrow \mathbf{C}$ be a one-to-one analytic map. We say that $f$ is WP-class if it has a quasiconformal extension to $\overline{\mathbf{C}}$ whose Beltrami differential $\mu$ on $\mathbf{D}^{-}$is in $L_{\text {hyp }}^{2}\left(\mathbf{D}^{-}\right)$. We say that a Jordan curve $\Gamma$ in $\mathbf{C}$ is a WP-class quasicircle if there is a WP-class map $f$ taking $\mathbf{D}^{+}$onto the bounded complement of $\Gamma$.

As above, we assume that $\infty \notin \Gamma$ for convenience throughout the paper, although the definition above can be naturally extended to allow this possibility. We refer to each of the two complements of a WP-class quasicircle as WP-class quasidisks.

We will also need the appropriate function spaces of boundary values corresponding to the Dirichlet problem. In the case of a WP-class quasidisks, the most natural space is a certain Besov space, which we will define shortly. To this end, let us recall the definition of an Ahlfors-regular set in $\mathbf{R}^{2}$.

Definition 2.4. Let $E$ be a compact subset of $\mathbf{R}^{2}$. One says that $E$ is Ahlforsregular (or 1-regular) if it is bounded and if there is a constant $C_{E}$ such that

$$
\frac{1}{C_{E}} r \leq \mathscr{H}(B(x, r) \cap E) \leq C_{E} r
$$

for all $x \in E, 0<r \leq \operatorname{diam}(E)$, where $\mathscr{H}$ denotes the one-dimensional Hausdorff measure.

From this, it also follows that the Hausdorff dimension $d_{\mathrm{H}}$ of an Ahlfors-regular set is equal to 1 . In this paper we will be particularly concerned with WP-class quasicircles which we later show are Ahlfors-regular, see Theorem 2.8.

We now define the relevant Besov space $\mathcal{H}(\partial \Omega)$ :

Definition 2.5. Let $\Omega \subset \mathbf{R}^{2}$ with an Ahlfors-regular boundary $\partial \Omega$. The Besov space $\mathcal{H}(\partial \Omega)$ consists of all $u \in L^{2}(\partial \Omega)$ for which

$$
\begin{aligned}
\|u\|_{\mathcal{H}(\partial \Omega)}:= & \left\{\int_{\partial \Omega}|u(x)|^{2} d \mathscr{H}(x)\right\}^{1 / 2} \\
& +\left\{\iint_{|x-y|<1} \frac{|u(x)-u(y)|^{2}}{|x-y|^{2}} d \mathscr{H}(x) d \mathscr{H}(y)\right\}^{1 / 2}<\infty .
\end{aligned}
$$

Note that in the case that $\partial \Omega=\mathbf{S}^{1}$ (the unit circle), this reduces to the Sobolev space $\mathcal{H}\left(\mathbf{S}^{1}\right)$ of functions with square-integrable half-order derivatives, see e.g. [12]. It was shown by Jonsson [11] that if $\Omega$ is a domain in $\mathbf{R}^{2}$ whose boundary $\partial \Omega$ is Ahlfors-regular, then the elements of $H^{1}(\Omega)$ have a well-defined trace or restriction to $\partial \Omega$. More precisely, given $\Omega \subset \mathbf{R}^{2}$ whose boundary $\partial \Omega$ is Ahlfors-regular and given $f \in H^{1}(\Omega)$, its trace $\left.f\right|_{\partial \Omega}$ exists as a function in the Besov space $\mathcal{H}(\partial \Omega)$, moreover for some $C>0$ one has $\left\|\left.f\right|_{\partial \Omega}\right\|_{\mathcal{H}(\partial \Omega)} \leq C\|f\|_{H^{1}(\Omega)}$. Conversely, every function $f \in \mathcal{H}(\partial \Omega)$ can be extended to a function $F \in H^{1}(\Omega)$ in such a way that $F$ depends continuously on the boundary data. 
From this and the discussion above on the regularity of the quasicircles, we can immediately conclude

Proposition 2.6. Given a WP-class quasidisk $\Omega$ and $u \in H^{1}(\Omega)$, one has $\left\|\left.u\right|_{\partial \Omega}\right\|_{\mathcal{H}(\partial \Omega)} \leq C\|u\|_{H^{1}(\Omega)}$. Conversely a function $u \in \mathcal{H}(\partial \Omega)$ has an extension to a function $U \in H^{1}(\Omega)$.

We also need to recall the definition of a chord-arc curve.

Definition 2.7. A closed curve in $\mathbf{R}^{2}$ is called chord-arc if it is an Ahlfors-regular quasicircle.

Therefore $\Gamma$ is a chord-arc curve if and only if it is rectifiable and there is a constant $C>0$ such that the length of the shorter arc of $\Gamma$ joining the two points $w_{1}$ and $w_{2}$ is bounded from above by $C\left|w_{1}-w_{2}\right|$ (see e.g. [8]).

The following theorem establishes the fact that WP-class quasidisks are chordarc domains, i.e., bounded domains in the plane that have a chord-arc boundary curve. This gives a partial answer to the problem of intrinsically characterizing WPclass quasidisks posed by Takhtajan and Teo [18, Part II Remark 1.10]. (In the same remark they also posed the problem of intrinsically characterizing WP-class quasisymmetries - solved by Shen [15]).

Theorem 2.8. Let the Beltrami differential $\mu$ belong to $L_{\text {hyp }}^{2}\left(\mathbf{D}^{+}\right)$. Then the corresponding WP-class quasicircle is a bi-Lipschitz image of the circle $\mathbf{S}^{1}$ and hence a chord-arc curve. Furthermore the corresponding WP-class quasidisk is a bi-Lipschitz image of the unit disk $\mathbf{D}^{+}$.

Proof. The assumption that the Beltrami coefficient $\mu \in L_{\text {hyp }}^{2}\left(\mathbf{D}^{+}\right)$and the isomorphism $1 / z$ between $\mathbf{D}^{+}$and $\mathbf{D}^{-}$together with a result of Becker and Pommerenke [3, Corollary 1.4] yield that the corresponding quasiconformal map $f^{\mu}$ is asymptotically conformal in the sense of [3]. From this and a result of Badger, Gill, Rohde and Toro (see [2, Corollary 2.7]) it readily follows that $d_{\mathrm{H}}\left(f^{\mu}\left(\mathbf{S}^{1}\right)\right)=1$, i.e., the quasicircle associated with $\mu \in L_{\text {hyp }}^{2}\left(\mathbf{D}^{+}\right)$has Hausdorff dimension exactly equal to 1 . At this point we use a classical result of Falconer and Marsh [7] that if two quasicircles have the same Hausdorff dimension then they are bi-Lipschitz homeomorphic. Now since $d_{\mathrm{H}}\left(\mathbf{S}^{1}\right)=1$ it follows that there exists a bi-Lipschitz map $\varphi$ from $S^{1}$ to $f^{\mu}\left(\mathbf{S}^{1}\right)=\varphi\left(\mathbf{S}^{1}\right)$. Now it follows from a result of Tukia [19] that $\varphi$ has a bi-Lipschitz extension to the plane. Furthermore, since $\mathbf{S}^{1}$ is both Ahlfors-regular and rectifiable and these two properties are both preserved under bi-Lipschitz homeomorphisms, it follows at once that $f^{\mu}\left(\mathbf{S}^{1}\right)$ is a chord-arc curve.

2.3. Solvability of the Dirichlet problem. Now, using the fact that a WPclass quasidisk is a chord-arc domain, one can show that the Dirichlet problem is solvable on a WP-class quasidisk $\Omega$ with boundary values in $\mathcal{H}(\partial \Omega)$. In fact we show that $\mathcal{H}(\partial \Omega)$ consists precisely of functions which are boundary values of harmonic functions in $\Omega$. This establishes its naturality.

Theorem 2.9. Let $\Omega$ be a WP-class quasidisk such that $\infty \notin \Omega$ and $0 \in \Omega$. Then the following statements are valid.

(1) Every function $h \in \mathcal{H}(\partial \Omega)$ is the trace of an element $H \in \mathcal{D}_{\text {harm }}(\Omega)$; furthermore, the linear operator taking $h$ to $H$ is bounded with respect to the $\mathcal{D}_{\text {harm }}(\Omega)$ and Besov norms. 
(2) Every element $H \in \mathcal{D}_{\text {harm }}(\Omega)$ has a trace in $\mathcal{H}(\partial \Omega)$. Furthermore the linear operator taking $H$ to its trace is bounded with respect to the $\mathcal{D}_{\text {harm }}$ norm and Besov norms.

Proof. (1) A careful examination of the proof of Theorem 3.4 in [13] reveals that the Dirichlet problem on a chord-arc domain with boundary data in $\mathcal{H}(\partial \Omega)$ has a solution whose $H^{1}$ norm depends continuously on the data; see in particular [4]. Since the $H^{1}$ and $\mathcal{D}_{\text {harm }}$ norms are equivalent by Theorem 2.2, this proves the first part of the theorem.

(2) The identity map from $\mathcal{D}_{\text {harm }}(\Omega)$ to $H^{1}(\Omega)$ is bounded by Theorem 2.2 . The claim now follows from the fact that the trace operator from $H^{1}(\Omega)$ to $\mathcal{H}(\partial \Omega)$ is bounded by Proposition 2.6.

It follows directly from Theorem 2.9 that composition by a conformally extendible map between quasicircles is a bounded invertible map between the Besov spaces.

Theorem 2.10. Let $\Omega_{1}$ and $\Omega_{2}$ be WP-class quasidisks containing 0 and such that $\infty \notin \partial \Omega_{i}$. If $F: \Omega_{1} \rightarrow \Omega_{2}$ is a conformal map taking 0 to 0 , and $C_{F}: \mathcal{H}\left(\partial \Omega_{2}\right) \rightarrow$ $\mathcal{H}\left(\partial \Omega_{1}\right)$ is composition by the trace of $F$ on the boundary (i.e., $\left.h \mapsto h \circ F\right)$, then $C_{F}$ is a bounded map.

Proof. First, observe that since $\Omega_{1}$ and $\Omega_{2}$ are quasidisks, $F$ has a continuous extension to $\partial \Omega_{1}$ which is a homeomorphism of $\Omega_{1}$ onto $\Omega_{2}$.

Let $h \in \mathcal{H}\left(\partial \Omega_{2}\right)$. By Theorem $2.9 H$ has a harmonic extension depending continuously on $h$. Now since $H \mapsto H \circ F$ is an isometry in $\mathcal{D}_{\text {harm }}(\Omega)$, Theorem 2.9 yields the claim.

Remark 2.11. Note $F$ extends to a quasisymmetry of the boundary. Since $C_{F^{-1}}=C_{F}^{-1}$, we have that $C_{F}$ has a bounded inverse under the hypotheses of the theorem.

Remark 2.12. Theorem 2.10 demonstrates that the Besov space $\mathcal{H}(\partial \Omega)$ inherits the property of conformal invariance from the harmonic Dirichlet space.

\section{Jump formula and Cauchy projections}

3.1. Solution of the jump problem on WP-class quasicircles. In this subsection, we show that the jump problem is solvable for WP-quasidisks for boundary values in $\mathcal{H}(\partial \Omega)$. From Theorem 2.8 it follows immediately that the boundary of a WP-class quasidisk is a rectifiable curve and therefore Cauchy integrals will be defined in a natural way on the WP-class quasicircles. Next we discuss Cauchy integrals. Let $\Gamma$ be a closed oriented rectifiable Jordan curve in the plane not containing $\infty$ and let $\Omega^{+}$and $\Omega^{-}$denote its two complementary regions. $\Omega^{-}$will denote the region containing $\infty$. Given a function $f$ on $\Gamma$ one defines its Cauchy integral $P(\Gamma) f(z)$ for $z \notin \Gamma$ by

$$
P(\Gamma) f(z)=\frac{1}{2 \pi i} \int_{\Gamma} \frac{f(\zeta)}{\zeta-z} d \zeta
$$

Now if $P_{+}(\Gamma) f$ and $P_{-}(\Gamma) f$ are restrictions of $P(\Gamma) f(z)$ to $\Omega^{+}$and $\Omega^{-}$respectively, and if $f_{+}$and $f_{-}$are their boundary values, the Sokhotski-Plemelj jump formula yields that

$$
f_{ \pm}(z)=\frac{ \pm 1}{2} f(z)+\frac{1}{2 \pi i} \mathrm{P} . \mathrm{V} \cdot \int_{\Gamma} \frac{f(\zeta)}{\zeta-z} d \zeta, \quad z \in \Gamma
$$


A classical result due to David [6] yields that if $\Gamma$ is a chord-arc curve then given $f \in L^{2}(\Gamma)$, one has the estimate, $\left\|f_{ \pm}\right\|_{L^{2}(\Gamma)} \leq C\|f\|_{L^{2}(\Gamma)}$.

We will also need estimates for a certain integral operator that appears frequently in function theory. This operator is defined by

$$
T_{\Omega} f(z)=\iint_{\Omega} \frac{f(\zeta)}{\zeta-z} d A(\zeta)
$$

We also have that

$$
\partial_{z} T_{\Omega} f(z)=\lim _{\varepsilon \rightarrow 0} \iint_{\Omega \cap\{|\zeta-z|>\varepsilon\}} \frac{f(\zeta)}{(\zeta-z)^{2}} d A(\zeta) .
$$

Lemma 3.1. Let $\Omega$ be a bounded domain in the plane. Then $\left\|T_{\Omega} f\right\|_{L^{2}(\Omega)} \leq$ $C\|f\|_{L^{2}(\Omega)}$ and $\left\|\partial_{z} T_{\Omega} f\right\|_{L^{2}(\Omega)} \leq C\|f\|_{L^{2}(\Omega)}$. Thus since $\partial_{\bar{z}} T_{\Omega} f=\pi f \chi_{\Omega}$ we have

$$
\left\|T_{\Omega} f\right\|_{H^{1}(\Omega)} \leq C\|f\|_{L^{2}(\Omega)} .
$$

Proof. To establish the boundedness of $T_{\Omega}$ on $L^{2}(\Omega)$, we observe that using the Cauchy-Schwarz inequality we have the pointwise estimate

$$
\left|T_{\Omega} f(z)\right| \leq\left\{\iint_{\Omega} \frac{|f(\zeta)|^{2}}{|\zeta-z|} d A(\zeta)\right\}^{1 / 2}\left\{\iint_{\Omega} \frac{1}{|\zeta-z|} d A(\zeta)\right\}^{1 / 2} .
$$

But if $|\Omega|$ denotes the 2-dimensional Lebesgue measure of $\Omega$, then it can be shown, see e.g., Tutschke [20] that $\iint_{\Omega} \frac{1}{|\zeta-z|} d A(\zeta) \leq 2 \sqrt{\pi}|\Omega|^{1 / 2}$, for $z \in \Omega$. Therefore squaring and integrating (3.6) and using Fubini's theorem, we obtain

$$
\iint_{\Omega}\left|T_{\Omega} f(z)\right|^{2} d A(z) \leq 4 \pi|\Omega|\|f\|_{L^{2}(\Omega)}^{2}
$$

which is the desired $L^{2}$ boundedness.

In order to show the $L^{2}(\Omega)$ boundedness of $\partial_{z} T_{\Omega}$ we just use (3.4) and the fact that

$$
\left\|\partial_{z} T_{\Omega} f\right\|_{L^{2}(\Omega)} \leq\left\|\partial_{z} T_{\Omega} f\right\|_{L^{2}(\mathbf{C})}=\left\|\partial_{z} T_{\mathbf{C}}\left(f \chi_{\Omega}\right)\right\|_{L^{2}(\mathbf{C})},
$$

where $\chi_{\Omega}$ denotes the characteristic function of $\Omega$. Now, as was shown by Ahlfors in [1], $\left\|\partial_{z} T_{\mathbf{C}} f\right\|_{L^{2}(\mathbf{C})}=\pi\|f\|_{L^{2}(\mathbf{C})}$. Therefore

$$
\left\|\partial_{z} T_{\Omega} f\right\|_{L^{2}(\Omega)} \leq \pi\left\|f \chi_{\Omega}\right\|_{L^{2}(\mathbf{C})}=\pi\|f\|_{L^{2}(\Omega)},
$$

which concludes the proof of the lemma.

Now we have all the ingredients to state and solve the following Riemann boundary value problem, sometimes called (with various kinds of regularity) the jump problem.

Theorem 3.2. Let $\Omega^{+}$be a WP-class quasidisk as above and let $u$ be in $\mathcal{H}\left(\partial \Omega^{+}\right)$. Let $\Omega^{-}$denote the complement of $\overline{\Omega^{+}}$in $\overline{\mathbf{C}}$. Then the jump problem with data $u$ can be solved in the sense that there exist holomorphic functions $u_{ \pm}$on $\Omega^{ \pm}$such that, $u_{ \pm} \in \mathcal{D}\left(\Omega^{ \pm}\right)$and $u_{+}(z)-u_{-}(z)=u(z)$ for $z \in \partial \Omega$. Furthermore $u_{ \pm}$depend continuously on the data; that is the Cauchy projections are bounded.

Proof. From the discussion above on the Cauchy integral, it readily follows that the solution of this problem is given by $u(z)_{ \pm}=P_{ \pm}\left(\partial \Omega^{+}\right) u(z)$. It remains to prove that one has the estimate

$$
\left\|u_{+}\right\|_{H^{1}\left(\Omega^{+}\right)} \leq c\|u\|_{\mathcal{H}\left(\partial \Omega^{+}\right)} .
$$

The corresponding estimate on $\Omega^{-}$is similar and hence omitted. Now since $u \in$ $\mathcal{H}\left(\partial \Omega^{+}\right)$, it has an extension $v \in H^{1}\left(\Omega^{+}\right)$, therefore Proposition 2.6 yields that 
$\|v\|_{H^{1}\left(\Omega^{+}\right)} \leq c\|u\|_{\mathcal{H}\left(\partial \Omega^{+}\right)}$. Moreover it is known that for $v \in H^{1}\left(\Omega^{+}\right)$(using the fact that $\partial \Omega^{+}$is rectifiable),

$$
P_{+}\left(\partial \Omega^{+}\right) v(z)=v(z)+\frac{1}{\pi} \iint_{\Omega^{+}} \frac{\bar{\partial} v(\zeta)}{\zeta-z} d A(\zeta)=v(z)+\frac{1}{\pi} T_{\Omega^{+}}(\bar{\partial} v)(z),
$$

where the integral above is taken as a principal value integral.

Using these facts and estimate (3.5) of Lemma 3.1 we can deduce that

$$
\begin{aligned}
\left\|u_{+}\right\|_{H^{1}\left(\Omega^{+}\right)} & =\left\|P_{+}\left(\partial \Omega^{+}\right) v\right\|_{H^{1}\left(\Omega^{+}\right)} \leq\|v\|_{H^{1}\left(\Omega^{+}\right)}+C_{1}\|\bar{\partial} v\|_{L^{2}\left(\Omega^{+}\right)} \\
& \leq\left(1+C_{1}\right)\|v\|_{H^{1}\left(\Omega^{+}\right)} \leq C_{2}\|u\|_{\mathcal{H}\left(\partial \Omega^{+}\right)}
\end{aligned}
$$

as claimed.

Now, as a corollary of Theorem 3.2 we have the following result.

Corollary 3.3. Let $\Omega^{+}$be a WP-class quasidisk in the plane such that $\infty \notin \overline{\Omega^{+}}$, bounded by the curve $\Gamma$. Then the operators $P_{ \pm}(\Gamma): \mathcal{H}(\Gamma) \rightarrow \mathcal{D}\left(\Omega^{ \pm}\right)$are bounded.

Note that because of the limiting behaviour of the Cauchy kernel as $z \rightarrow \infty$, we have that $P_{-}(\Gamma) h(z) \rightarrow 0$ as $z \rightarrow \infty$, so $P_{-}(\Gamma)$ does map into $\mathcal{D}\left(\Omega^{-}\right)$.

\section{References}

[1] Ahlfors, L.: Lectures on quasiconformal mappings. - Van Nostrand Mathematical Studies 10, D. Van Nostrand Co., Inc., Toronto, Ont.-New York-London, 1966.

[2] Badger, M., J. Gill, S. Rohde, and T. Toro: Quasisymmetry and rectifiability of quasispheres. - Preprint, arXiv:1201.1581.

[3] Becker, J., and Ch. Pommerenke: Über die quasikonforme Fortsetzung schlichter Funktionen. - Math. Z. 161:1, 1978, 69-80 (in German).

[4] Chang, T.-K., and J. Lewis: Boundary integral operators and boundary value problems for Laplace's equation. - Ark. Mat. 49:2, 2011, 239-276.

[5] Cui, G.: Integrably asymptotic affine homeomorphisms of the circle and Teichmüller spaces. Sci. China Ser. A 43:3, 2000, 267-279.

[6] David, G.: Opérateurs d'intégrale singulière sur les surfaces régulières. - Ann. Sci. Ećole Norm. Sup. (4) 21:2, 1988, 225-258.

[7] FALCONER, K., and D. MARsh: Classification of quasi-circles by Hausdorff dimension. - Nonlinearity 2:3, 1989, 489-493.

[8] Garnett, J., and D. Marshall: Harmonic measure. - New Math. Monogr. 2, Cambridge Univ. Press, Cambridge, 2008.

[9] Guo, H.: Integrable Teichmüller spaces. - Sci. China Ser. A 43:1, 2000, 47-58.

[10] Huang, Y.-Z.: Two-dimensional conformal geometry and vertex operator algebras. - Progr. Math. 148, Birkhäuser Boston, Inc., Boston, MA, 1997.

[11] Jonsson, A.: Besov spaces on closed subsets of $\mathbf{R}^{n}$. - Trans. Amer. Math. Soc. 341:1, 1994, $355-370$.

[12] Jonsson, A., and H. Wallin: Function spaces on subsets of $\mathbf{R}^{n}$. - Math. Rep. 2:1, 1984.

[13] Mitrea, D., M. Mitrea, and S. Monniaux: Weighted Sobolev space estimates for a class of singular integral operators. - In: Around the research of Vladimir Maz'ya III, Int. Math. Ser. (N. Y.) 13, Springer, New York, 2010, 179-200.

[14] Shen, Y.: On Grunsky operator. - Sci. China Ser. A 50:12, 2007, 1805-1817.

[15] Shen, Y.: Weil-Petersson Teichmüller space. - Prepint, arXiv:1304.3197.

[16] Smith, W., and D. Stegenga: Hölder domains and Poincaré domains. - Trans. Amer. Math. Soc. 319:1, 1990, 67-100. 
[17] Stanoyevitch, A., and D. Stegenga: Equivalence of analytic and Sobolev Poincaré inequalities for planar domains. - Pacific J. Math. 178:2, 1997, 363-375.

[18] Takhtajan, L., and L. P. Teo: Weil-Petersson metric on the universal Teichmüller space. Mem. Amer. Math. Soc. 183:861, 2006.

[19] Tukia, P.: Extension of quasisymmetric and Lipschitz embeddings of the real line into the plane. - Ann. Acad. Sci. Fenn. Ser. A I Math. 6:1, 1981, 89-94.

[20] Tutschke, W.: Partielle komplexe Differentialgleichungen in einer und in mehreren komplexen Variablen. Mit losen Berichtigungen. - Hochschulbücher für Mathematik 82, VEB Deutscher Verlag der Wissenschaften, Berlin, 1977.

Received 9 March 2015 • Accepted 2 June 2015 